\title{
Behavioral Recovery and Early Decision Making in Patients with Prolonged Disturbance in Consciousness after Traumatic Brain Injury
}

\author{
Joseph T. Giacino, ${ }^{1-3}$ Mark Sherer,,5 Andrea Christoforou,,2 Petra Maurer-Karattup, \\ Flora M. Hammond, ${ }^{7,8}$ David Long, ${ }^{9}$ and Emilia Bagiella ${ }^{10}$
}

\begin{abstract}
The extent of behavioral recovery that occurs in patients with traumatic disorders of consciousness (DoC) following discharge from the acute care setting has been under-studied and increases the risk of overly pessimistic outcome prediction. The aim of this observational cohort study was to systematically track behavioral and functional recovery in patients with prolonged traumatic DoC following discharge from the acute care setting. Standardized behavioral data were acquired from 95 patients in a minimally conscious (MCS) or vegetative state (VS) recruited from 11 clinic sites and randomly assigned to the placebo arm of a previously completed prospective clinical trial. Patients were followed for 6 weeks by blinded observers to determine frequency of recovery of six target behaviors associated with functional status. The Coma Recovery Scale-Revised and Disability Rating Scale were used to track reemergence of target behaviors and assess degree of functional disability, respectively. Twenty percent (95\% confidence interval [CI]: 13-30\%) of participants (mean age 37.2; median 47 days post-injury; 69 men) recovered all six target behaviors within the 6 week observation period. The odds of recovering a specific target behavior were 3.2 (95\% CI: 1.2-8.1) to 7.8 (95\% CI: 2.7-23.0) times higher for patients in MCS than for those in VS. Patients with preserved language function ("MCS+") recovered the most behaviors $(p \leq 0.002)$ and had the least disability $(p \leq 0.002)$ at follow-up. These findings suggest that recovery of high-level behaviors underpinning functional independence is common in patients with prolonged traumatic DoC. Clinicians involved in early prognostic counseling should recognize that failure to emerge from traumatic DoC before 28 days does not necessarily portend unfavorable outcome.
\end{abstract}

Keywords: consciousness; MCS; outcome research; TBI; VS

\section{Introduction}

M ONITORING BEHAVIORAL RECOVERY in patients who develop disorders of consciousness (DoC) after severe traumatic brain injury (TBI) is an essential component of diagnostic and prognostic assessment. Following emergence from coma, patients transition into the vegetative state (VS) or the minimally conscious state (MCS). In VS, sleep-wake cycles are restored but there is no behavioral evidence of awareness. ${ }^{1}$ In MCS, there is at least one definitive behavioral sign of conscious awareness. ${ }^{2}$ The diagnosis of "MCS+" (v. "MCS-") is made when the features of MCS include behavioral evidence of language comprehension or expression. ${ }^{3}$ This dichotomy is supported by functional neuroimaging evidence of language network activation following exposure to verbal instructions in patients who meet diagnostic criteria for MCS. ${ }^{4,5}$ Emergence from MCS is established when there are reliable yes-no responses to questions or reproducible instances of appropriate object use. ${ }^{2}$ There is also evidence that re-emergence of specific behaviors foreshadows subsequent cognitive and functional recovery. ${ }^{6-11}$

\footnotetext{
${ }^{1}$ Spaulding Rehabilitation Hospital, Charlestown, Massachusetts.

${ }^{2}$ Department of Physical Medicine and Rehabilitation, Harvard Medical School, Boston, Massachusetts.

${ }^{3}$ JFK Johnson Rehabilitation Institute, Edison, New Jersey.

${ }^{4}$ Methodist Rehabilitation Center, Jackson, Mississippi.

${ }^{5}$ TIRR Memorial Hermann, Houston, Texas.

${ }^{6}$ SRH Fachkrankenhaus Neresheim, Neresheim, Germany.

${ }^{7}$ Department of Physical Medicine and Rehabilitation, Carolinas Rehabilitation, Charlotte, North Carolina.

${ }^{8}$ Department of Physical Medicine and Rehabilitation, Indiana University School of Medicine, Indianapolis, Indiana.

${ }^{9}$ Brain Injury Program, Bryn Mawr Rehab Hospital, Malvern, Pennsylvania.

${ }^{10}$ Center for Biostatistics, Icahn School of Medicine at Mount Sinai, New York, New York.

(c) Joseph T. Giacino et al., 2019; Published by Mary Ann Liebert, Inc. This Open Access article is distributed under the terms of the Creative Commons Attribution Noncommercial License (http://creativecommons.org/licenses/by-nc/4.0/) which permits any noncommercial use, distribution, and reproduction in any medium, provided the original author(s) and the source are credited.
} 
Prognostic decision making in the intensive care setting relies heavily on clinical findings that can be obtained within the first 7 days post-injury. Early prognostic indicators typically include demographics (age, sex), markers of clinical severity (Glasgow Coma Scale [GCS] score, pupillary reactivity), secondary insults (hypoxia, hypotension), computed tomographic (CT) characteristics, laboratory findings, and vital signs (blood pressure). The widely used International Mission for Prognosis and Analysis of Clinical Trials in TBI (IMPACT) model ${ }^{12}$ combines these characteristics to derive a cumulative score that predicts unfavorable outcome, defined as a Glasgow Outcome Scale ${ }^{13}$ rating $<4$ (i.e., fully dependent). Although the IMPACT model empirically optimizes early prognostication, its accuracy in discriminating unfavorable and favorable outcomes is $\sim 0.80$, suggesting that it should not be applied directly for treatment-limiting decisions. Consequently, outcome prediction rests largely on clinical judgement, which may lead to underestimation of recovery potential and failure to recommend aggressive treatment. A retrospective study of 720 adults with TBI hospitalized at six Level 1 Canadian trauma centers found that $70 \%$ of deaths in the intensive care unit (ICU) were related to withdrawal of life-sustaining therapy. In nearly $65 \%$ of these cases, the decision to withdraw treatment was made within 3 days of admission. ${ }^{14}$ Behaviors that presage later recovery may not emerge within this truncated time frame, contributing to premature treatment withdrawal. In fact, the major factor driving the decision to discontinue life support in the Canadian study (29\% of cases), was the clinician's opinion that the prognosis for neurological recovery was poor. The incidence of severe TBI is highest between the ages of 16 and $35,{ }^{15}$ placing young adults at the highest risk. It is clear that attitudinal variables influence decisions concerning treatment intensity and mortality rates ${ }^{16,17}$ among patients with severe TBI.

We previously reported the results of a randomized controlled trial testing the effectiveness of amantadine hydrochloride in patients with prolonged ( $\geq 4$ weeks) traumatic DoC. ${ }^{8}$ Although we found that amantadine significantly accelerated the pace of recovery, we also observed that the placebo group demonstrated substantial recovery and achieved the same level of function as the amantadine group within 2 weeks of treatment discontinuation. In view of the degree of improvement we observed in the placebo group, we conducted retrospective analyses of the original data set to more fully characterize "late" behavioral and functional recovery in this population. Our primary objectives were to determine the frequency of recovery of six "target" behaviors that underpin functional independence, and assess the relationship between the number of behaviors recovered and degree of disability at follow-up. We were also interested in exploring differences in behavioral and functional outcome between patients diagnosed with MCS+, MCS-, and VS.

\section{Methods}

\section{Participants}

Participants were patients with traumatic DoC enrolled in the placebo arm of a prospective 6 week multi-center randomizedcontrolled trial investigating the effectiveness of amantadine hydrochloride in promoting recovery. ${ }^{8}$ Recruitment was conducted at eight rehabilitation hospitals in the United States and three in Europe between February 2005 and March 2010. All patients were in VS or MCS at enrollment. Of the 184 patients who completed the study, 97 were randomized to the placebo arm ( 35 in $\mathrm{VS}^{18}$ and 62 in $\mathrm{MCS}^{2}$ ). Two patients who were incorrectly assigned to the MCS stratum in the original study were reassigned to the VS stratum for the current analyses. Participants were 16-65 years of age, had sustained a non-penetrating TBI 4-16 weeks prior to enrollment, underwent withdrawal of psychoactive medications, and received the usual inpatient rehabilitation services. Patients with pre-injury central nervous system-related disability, medical instability, pregnancy, more than one seizure in the prior month, and prior amantadine treatment were excluded. Additional eligibility details and the Consolidated Standards of Reporting Trials (CONSORT) diagram are described in the original publication. ${ }^{8}$ Patients were stratified by length of time since injury (early $=28-71$ days; late $=72-112$ days) to investigate the effects of injury chronicity on behavioral recovery. ${ }^{19}$ Patients in MCS were sub-stratified into MCS+ or MCS- subgroups, based on performance on language items from the Coma Recovery Scale - Revised (CRS-R) ${ }^{20}$ (see Fig. S1 and Table S1).

\section{Outcomes}

Outcomes were assessed using the CRS- $\mathrm{R}^{20}$ and Disability Rating Scale (DRS). ${ }^{21}$ The CRS-R is composed of six subscales designed to assess level of consciousness and track behavioral recovery (Fig. S1). ${ }^{20,22}$ Reliability and validity are well established, ${ }^{23}$ supporting its selection by the National Institute on Neurological Disorders and Stroke TBI Common Data Element Workgroup as the preferred measure for monitoring recovery of consciousness. ${ }^{24}$ We chose the most complex behavior assessed on each CRS-R subscale to serve as "target" behaviors. The six behaviors selected and their definitions are shown in Table 1. Emergence from MCS was demonstrated by either reliable yes-no responses to questions (i.e., CRS-R Communication subscale score of 2) or reproducible instances of appropriate object use (i.e., CRS-R Motor subscale score of 6). ${ }^{2}$ The DRS is a TBI-specific functional outcome scale that includes the GCS; ${ }^{25}$ ratings of cognitive ability for feeding, dressing, and grooming; degree of assistance required; and employability. Scores range from 0 to 29 , with higher values indicating greater disability (Table S2). Scores were grouped into disability categories to describe outcomes at week $6 .{ }^{8}$

\section{Procedure}

Study staff who were responsible for administering the CRS-R attended a pre-enrollment training seminar taught by the primary author of the CRS-R (J.T.G.), and were required to utilize the CRS$\mathrm{R}$ Administration and Scoring Manual when conducting study examinations. The CRS-R was administered at enrollment and at the 6 week follow-up to track recovery of the target behaviors. DRS scores were obtained independently by consensus of the rehabilitation team at week 6 and were used to investigate the relationship between number of behaviors recovered and degree of functional disability. The number of patients who emerged from MCS at week 6 was determined based on the CRS-R score profile. All patients completed the study within 22 weeks of injury.

The protocol was approved by the Institutional Review Boards of participating sites, written informed consent obtained from the legally authorized representative, and the trial was registered with ClinicalTrials.gov (ID\# NCT00970944). Independent oversight was provided by a data safety monitoring board and data were stored and analyzed by a data coordinating center.

\section{Statistical analysis}

We used descriptive statistics to summarize the demographic and clinical characteristics of the entire sample and subgroups stratified by time from injury and diagnosis. Two patients failed to complete the follow-up and were excluded from week 6 analyses, reducing the number of MCS patients from 62 to 60 . We calculated the proportions of patients (and exact 95\% confidence intervals [CI]) in the sample who recovered each target behavior and the total 
Table 1. Operational Definitions of the Six Target Behaviors ${ }^{\mathrm{a}}$

Target behavior

Consistent command following

Object recognition

Functional object use

in 2 of 2 trials with two different objects.

Intelligible verbalization Two different fully intelligible words consisting of a consonant-vowel-consonant blend must be detected during the course of the examination.

Reliable communication Clearly discernible and accurate verbal or gestural yes-no responses occur on 6 of 6 questions concerning situational orientation (e.g., "Am I touching my ear/nose?").

Sustained attention $\quad$ No more than 3 instances of failure to respond to a verbal prompt occur during CRS-R administration.

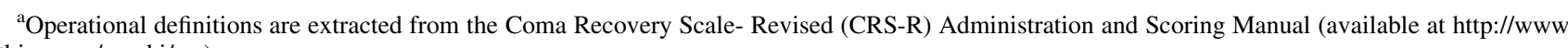
.tbims.org/combi/crs)

${ }^{\mathrm{b}} \mathrm{A}$ scoreable response occurs when the target behavior is observed within $10 \mathrm{sec}$ of the examiner's standardized prompt.

number of behaviors recovered by week 6 . To assess the influence of time post-injury on behavioral recovery, Fisher's Exact Tests (FET) and odds ratios (with 95\% CI) or the Mantel-Haenszel $\chi^{2}$ trend test were used to contrast recovery rates at week 6 between the early and late enrollment subgroups. We conducted the same analyses to compare recovery between conscious (MCS+ and MCS-) and unconscious (VS) patients. For the comparison between patients with and without preserved language function (MCS+ vs. MCS- and VS), we used Mantel-Haenszel $\chi^{2}$ analysis to compare the number of behaviors recovered at week 6. Mantel-Haenszel $\chi^{2}$ analysis was also used to determine if there were differences in the frequency of emergence from MCS. We also investigated the influence of age on recovery by comparing the mean age of patients who did and did not recover individual target behaviors by week 6 , and by comparing age distributions for the number of target behaviors recovered by week 6 .

To assess the relationship between behavioral recovery and functional disability, we calculated the median DRS score relative to the number of behaviors present at week 6 . We also performed linear regression analysis of the number of behaviors recovered at week 6 against the DRS score. To explore differences in functional outcome at week 6 between patients with and without language function at enrollment, we compared DRS scores using the Kruskal-Wallis test. The Kruskal-Wallis test was also used in a post-hoc analysis to determine if the MCS+ and MCS- subgroups differed in length of time post-injury. We used Holm's Sequential Bonferroni Procedure ${ }^{26}$ to adjust for multiple comparisons, and considered results to be significant at $p \leq 0.05$. Analyses were performed using SAS, version 9.4 (SAS Institute, Inc.).

\section{Results}

Demographic and clinical characteristics of the sample are detailed in Table 2. Seventy-one percent of the sample was male, and the median time from injury to enrollment was 47 days (interquartile range $[\mathrm{IQR}]=37-65$ ). The mean CRS-R total score was 9.2 (standard deviation $[\mathrm{SD}]=4.4$ ) and the mean DRS rating was 22.2 $(\mathrm{SD}=2.1)$. There were no significant differences in age, sex or time from injury to enrollment (i.e., early, late) among the three diagnostic strata (MCS+, MCS-, VS).

The proportion of patients in the total sample demonstrating each target behavior at enrollment and week 6 is depicted in Figure 1A. At enrollment, 1 patient in MCS demonstrated two behaviors, 10 demonstrated one, and the remainder of the sample demonstrated none. By week 6, there was a marked increase in the prevalence of the six target behaviors, ranging from $29 \%(95 \%$ $\mathrm{CI}=20-39 \%)$ for reliable yes-no communication to $42 \%(95 \%$ $\mathrm{CI}=32-53 \%$ ) for object recognition. Nearly one third of the sample was communicating reliably by week 6 , signaling emergence from MCS. The only difference in behavioral recovery between patients enrolled early and those enrolled late was for intelligible speech, which was observed more often at week 6 in those enrolled early $(p=0.02$; odds ratio $[\mathrm{OR}]=8.8 ; 95 \%$ CI: $1.9-40.4)$ (Table S3). Patients who recovered target behaviors by week 6 were $2-7$ years younger on average than those who failed to do so (Table S4). The one exception was intelligible speech; patients who recovered this behavior were $\sim 3$ years older than those who did not.

We found a bimodal pattern for the number of target behaviors demonstrated at week 6 . Although $42 \%(95 \% \mathrm{CI}=32-53 \%)$ of the sample failed to recover any behaviors by week 6, 20\% (95\%

Table 2. Demographic and Clinical Characteristics AT ENROLLMENT

\begin{tabular}{lc} 
Characteristic & $\begin{array}{c}\text { Total sample } \\
(\mathrm{n}=97)\end{array}$ \\
\hline Age, mean (SD), y & $37.2(15.4)$ \\
No. (\%) Male & $69(71)$ \\
Time from injury, median (IQR), days & $47(37-65)$ \\
Coma Recovery Scale-Revised, mean (SD) ${ }^{\mathrm{a}}$, score & $9.2 \pm 4.4$ \\
Disability Rating Scale, mean (SD) ${ }^{\mathrm{b}}$, score & $22.2 \pm 2.1$ \\
No. (\%) in minimally conscious state "plus (+)" & $27(28)$ \\
No. (\%) in minimally conscious state "minus (-)" & $35(36)$ \\
No. (\%) in vegetative state & $35(36)$ \\
No. (\%) enrolled "early" (i.e., 28-71 days) & $77(79)$ \\
$\quad$ post-injury & $20(21)$ \\
No. (\%) enrolled "late" (i.e., 72-112 days) & \\
$\quad$ post-injury & \\
\hline
\end{tabular}

${ }^{\text {a }}$ Scores on the Coma Recovery Scale-Revised (CRS-R) ranged from 0 to 23 , with higher scores indicating a higher level of neurobehavioral function. CRS-R scores ranged from 2 to 19 at enrollment.

${ }^{\mathrm{b}} \mathrm{Scores}$ on the Disability Rating Scale (DRS) ranged from 0 to 29, with higher scores indicating more severe disability. DRS scores ranged from 17 to 28 at enrollment.

$\mathrm{SD}$, standard deviation; IQR, interquartile range. 


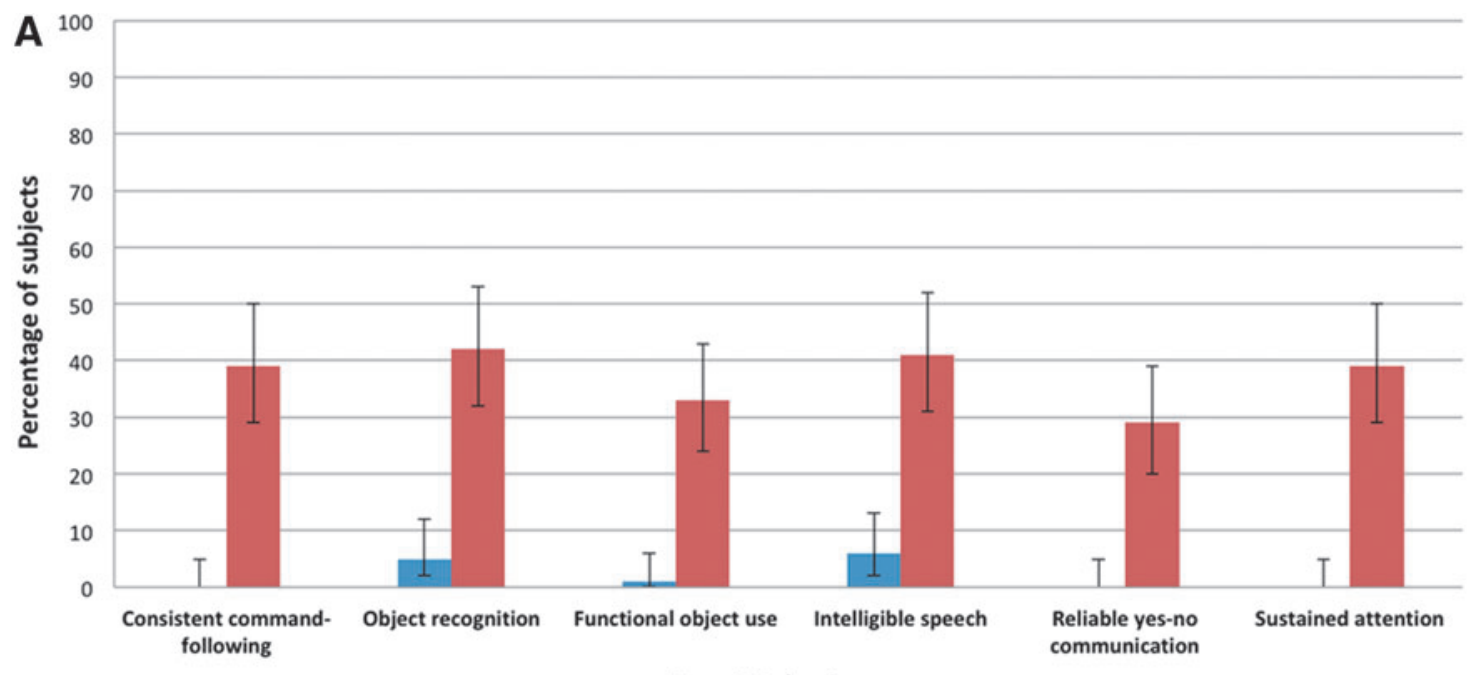

Target Behaviors

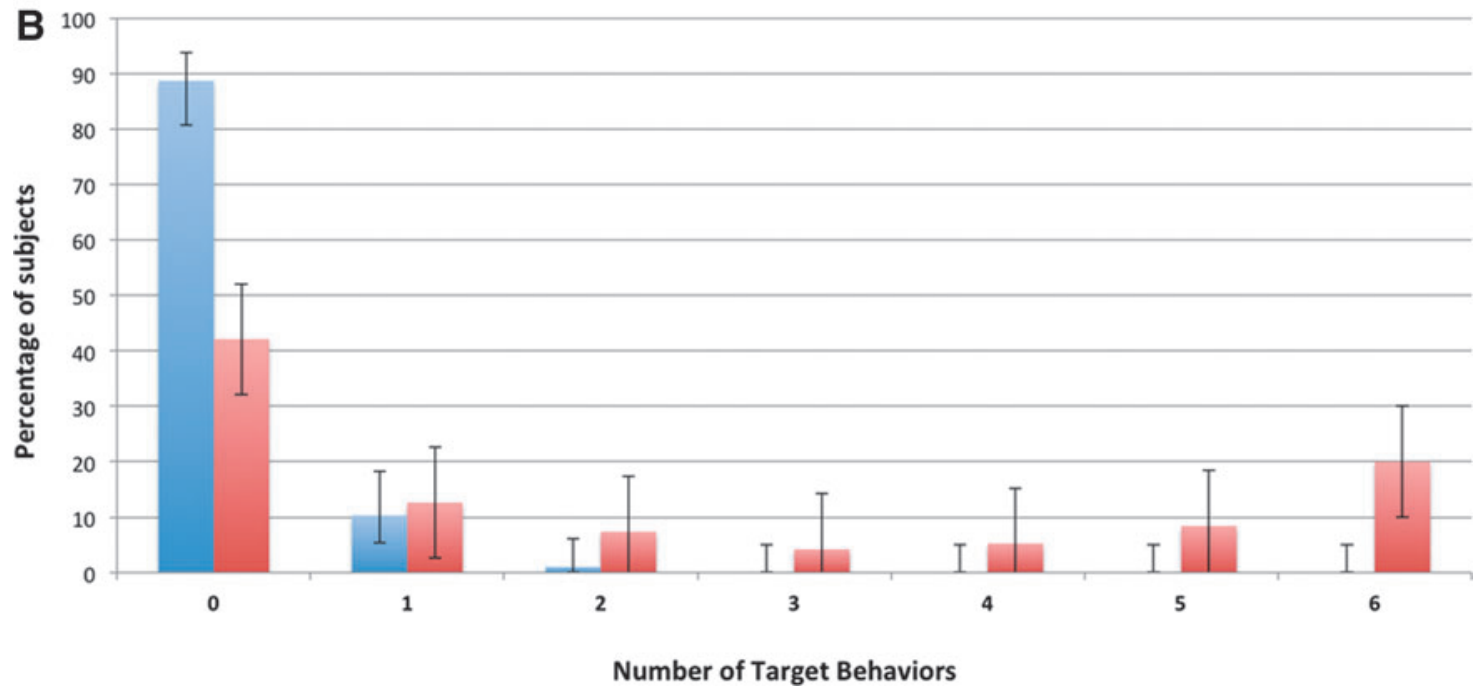

FIG. 1. Behavioral recovery rates among the total sample. (A) Bars indicate the proportion (and 95\% confidence interval) of participants among the total sample who demonstrated individual target behaviors at enrollment and at the week 6 follow-up. The six target behaviors were rarely observed at enrollment (blue bars). By week 6 (red bars), each individual target behavior was observed in $29-42 \%$ of the sample. (B) Bars show the proportion (and 95\% confidence interval) of participants who demonstrated zero to six target behaviors at enrollment (blue bars) and at week 6 (red bars). Although target behaviors were absent in nearly 9 of 10 participants at enrollment (blue bars), by week 6 (red bars), $>50 \%$ had recovered at least one target behavior.

$\mathrm{CI}=13-30 \%$ ) recovered all six (Fig. 1B and Table S5). Among patients who recovered at least five behaviors, 35\% (95\% CI: $25-47 \%$ ) were enrolled early and 5\% (95\% CI: $0.3-27 \%)$ were enrolled late, a non-significant trend ( $p=0.12$, Table S5). Age did not influence the number of target behaviors recovered by week 6 . The mean age of patients who recovered all six target behaviors (mean $=36.9, \mathrm{SD}=16.2$ ) was only slightly $>1$ year younger than those who recovered none $($ mean $=38.1, \mathrm{SD}=15.1)$ (Table S6).

At week 6, nearly three quarters $(74 \%)$ of the patients who recovered all six target behaviors had moderate to severe disability on the DRS. Conversely, $68 \%$ of patients who recovered no behaviors fell in the vegetative to extreme vegetative range (Table S7). The median DRS at week 6 was 11 (moderately-severe; IQR = 8-14) for patients who recovered six behaviors, 18 (extremely severe; $\mathrm{IQR}=14-21$ ) for those who recovered three, and 23 (vegetative to extreme vegetative state; $I Q R=21-24.5)$ for those who recovered none (Table S8). Regression analysis demonstrated a linear relationship between behavioral recovery and degree of disability (adjusted $R^{2}=0.64 ; p=0.002$ ). For each behavior recovered, the DRS score decreased (i.e., improved) by an average of 1.7 points.

Behavioral recovery rates differed between patients who were in MCS at enrollment and those in VS (Fig. 2, top panel). MCS recovery rates ranged from $40 \%$ (95\% CI: $28-53 \%$ ) for reliable communication to $57 \%$ (95\% CI: 43-69\%) for intelligible speech and object recognition. For VS, rates ranged from $11 \%$ (95\% CI: 4$28 \%$ ) for communication, to $23 \%$ (95\% CI: $11-41 \%$ ) for sustained attention. The odds of recovering a target behavior during the 6 week observation period were significantly higher for patients in MCS across five of the six behaviors (command following: $\mathrm{OR}=4.0,95 \%$ $\mathrm{CI}=1.5-10.6, p=0.04$; object recognition: $\mathrm{OR}=6.3,95 \% \mathrm{CI}=2.3-$ 


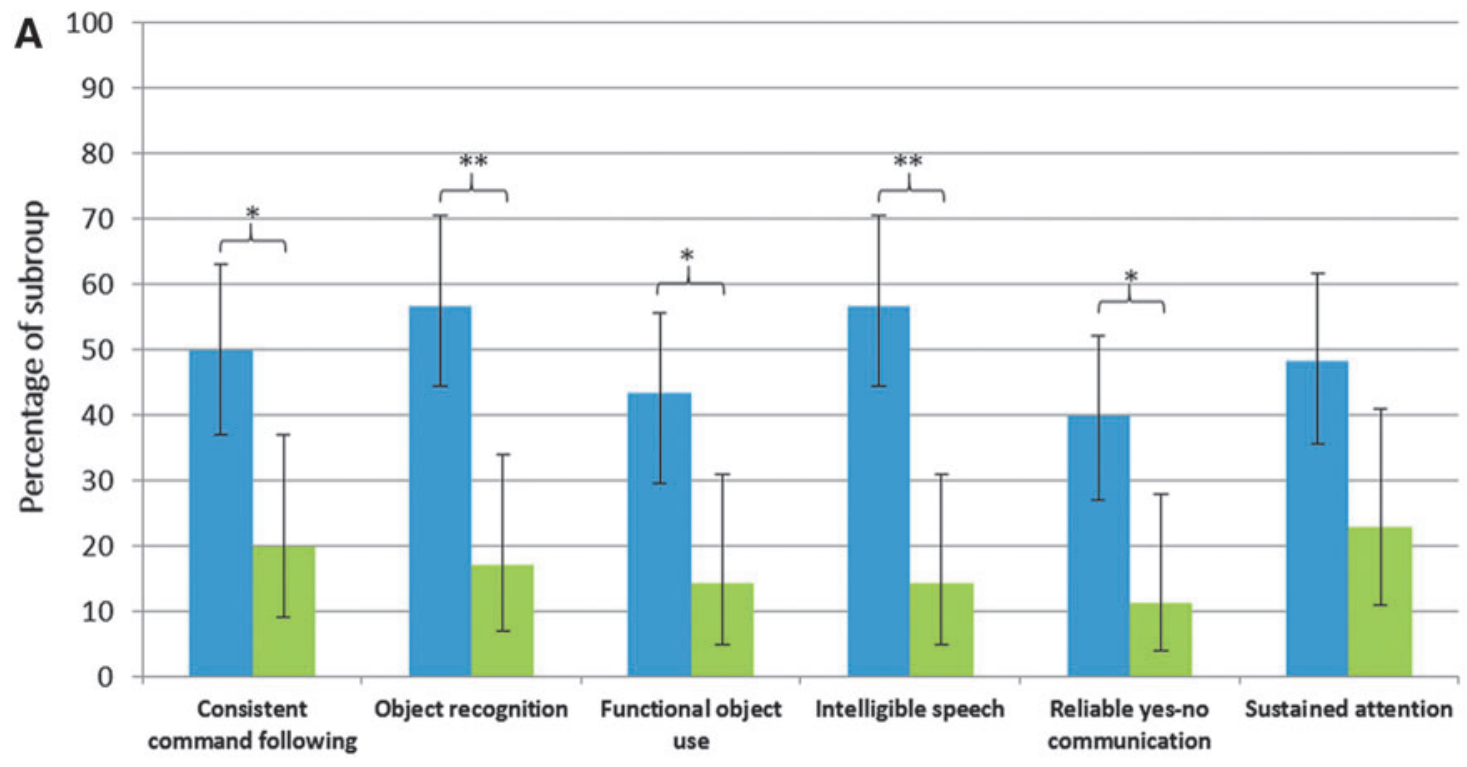

Target Behaviors

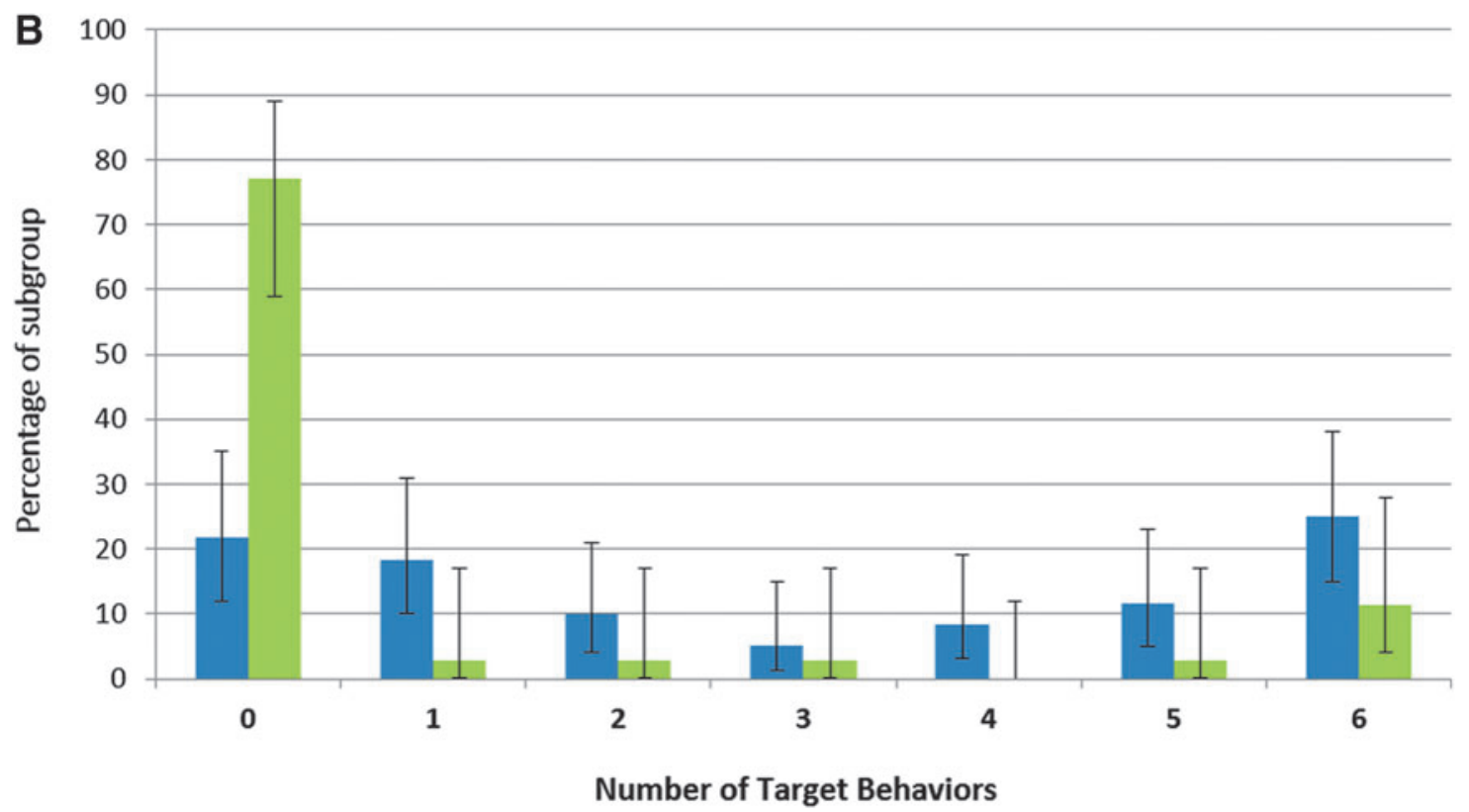

FIG. 2. Comparison of behavioral recovery between participants in a minimally conscious state (MCS) and and those in a vegetative state (VS). (A) Bars depict the individual behavioral recovery rates (proportion of subjects and 95\% confidence interval [CI]) for participants in MCS ( $n=60$, blue) and VS ( $n=35$, green) at week 6. Recovery rates were significantly higher for patients in MCS across five of the six target behaviors $(* p \leq 0.05 ; * * p \leq 0.01$ ). Odds ratios (with CIs) are shown in Supplementary Table S9. (B) Bars indicate the total number of target behaviors demonstrated in the MCS (blue) and VS (green) subgroups at the week 6 follow-up (proportion of subjects and 95\% CI). Participants enrolled in MCS recovered significantly more target behaviors by week 6 than those who were in VS $\left(\chi_{\mathrm{MH}}^{2}=13.9,1 d f ; p=0.0026\right)$.

17.5, $p=0.003$; functional object use: $\mathrm{OR}=4.6,95 \% \mathrm{CI}=1.6-13.4$, $p=0.04$; intelligible speech: $\mathrm{OR}=7.8,95 \% \mathrm{CI}=2.7-23.0, p=0.001$; reliable communication: $\mathrm{OR}=5.2,95 \% \mathrm{CI}=1.6-16.5, p=0.04$, Table S9). Sustained attention did not differ between these subgroups $(p=0.12)$. The difference in recovery rates between the two subgroups remained significant when we reran the analyses after removing MCS cases that had any of these behaviors at enrollment. At week 6, the total number of target behaviors recovered was also significantly higher in the MCS $\operatorname{subgroup}\left(\chi_{\mathrm{MH}}^{2}=13.9,1\right.$ $d f ; p=0.003$ ), with $37 \%$ recovering at least five behaviors as compared with $14 \%$ in the VS group (Fig. 2; Table S10). The odds of emerging from MCS were almost seven times higher for patients in $\mathrm{MCS}$ versus those in $\mathrm{VS}(\mathrm{MCS}=53 \%, \mathrm{VS}=14 \%$; $\mathrm{OR}=6.8,95 \% \mathrm{CI}$ : 2.3-20.1, $p=0.002$ ).

When we compared patients who showed preserved language function at enrollment (MCS+) with those who did not (MCS- and VS), we found that the MCS+ subgroup recovered significantly more target behaviors by week $6\left(\chi_{\mathrm{MH}}^{2}=21.9,1 d f ; p=0.002\right)$. 


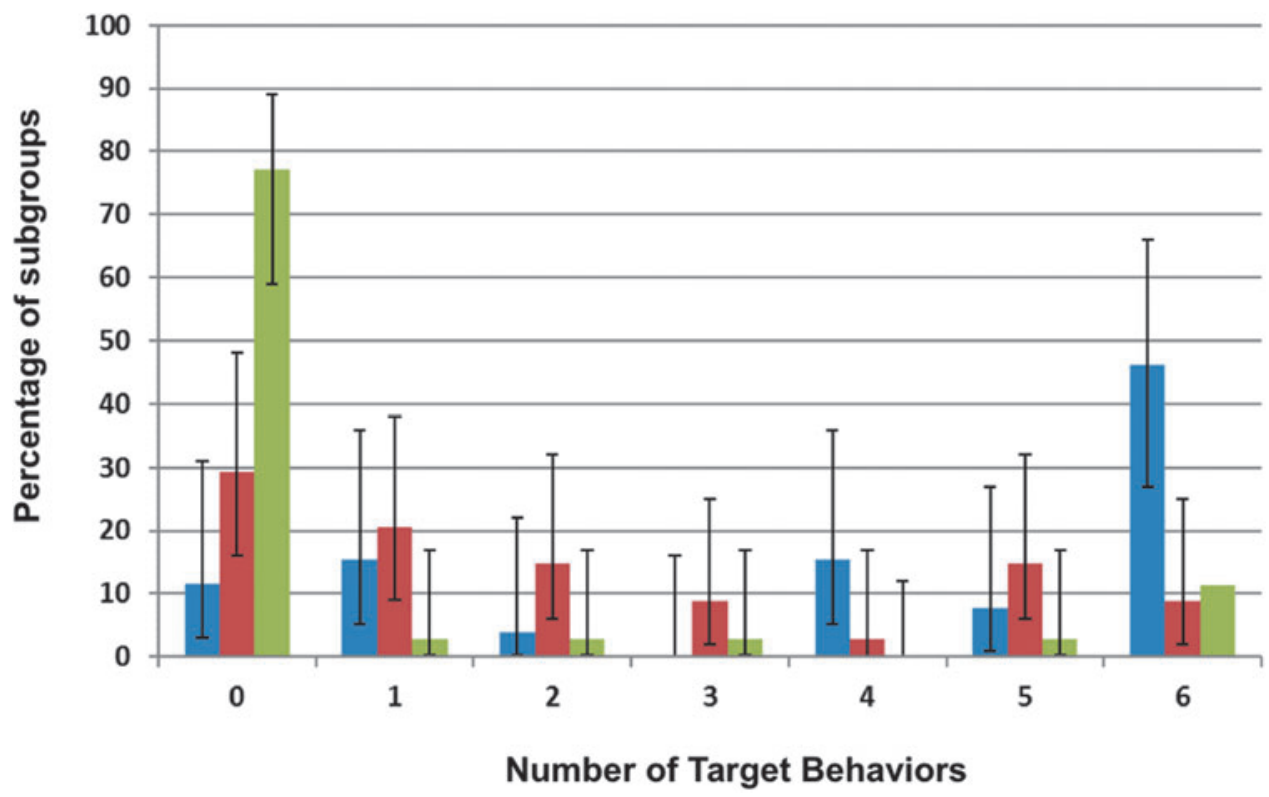

FIG. 3. Comparison of the total number of target behaviors recovered among participants with and without language function at enrollment. Bars show the proportion of participants (and 95\% confidence interval) in a minimally conscious state with preserved language function at enrollment (MCS +$)(n=26$, blue), in MCS without language function (MCS-) $(n=34$, red) and in a vegetative state (VS) ( $n=35$, green) against the total number of target behaviors recovered by week 6 . Participants with preserved language function at enrollment (MCS +$)$ recovered significantly more target behaviors than those in MCS- or VS $\left(\chi^{2} \mathrm{MH}=21.2,1 d f\right.$; $p \leq 0.0018)$.

Nearly half the MCS+ subgroup (46\%, 95\% CI: 27-66\%) recovered all six target behaviors as compared with 1 in 10 patients in the MCS- subgroup (9\%, 95\% CI: $2-25 \%)$ or the VS group (11\%, $95 \%$ CI: $3-27 \%$ ) (Fig. 3 and Table S11). At week 6, the proportion of patients who emerged from MCS $(\mathrm{MCS}+=73 \%$; MCS- $=38 \%$; $\left.\mathrm{VS}=14 \% ; \chi_{\mathrm{MH}}^{2}=21.2,1 d f ; p=0.002\right)$ and the median degree of disability on the DRS (MCS+: mean $=12$, IQR $=9-17$; MCS-: mean $=17, \mathrm{IQR}=15-19$; VS: mean $=23, \mathrm{IQR}=20-24 ; p=0.002$ ) were also more favorable in the MCS+ subgroup. Post-hoc analysis confirmed that these differences were not attributable to greater time from injury, as the median interval was shorter in the MCS+ subgroup $(\mathrm{MCS}+$ : mean $=38$ days, $\mathrm{IQR}=33-47$; MCS-: mean $=54$ days, IQR $=40-69$; Kruskal-Wallis Test $p<0.001$ ).

\section{Discussion}

Prognostication in patients with traumatic DoC depends largely on behavioral findings obtained through clinical examination. However, the frequency and time course of recovery of specific behaviors has not been well characterized. We systematically assessed rehabilitation inpatients with prolonged DoC over a 6 week period using a validated neurobehavioral assessment measure, the CRS-R. We tracked the frequency of recovery of six behaviors that represent precursors to functional independence and found that consistent command following, object recognition, functional object use, intelligible speech, yes-no communication, and sustained attention all commonly recover after 4 weeks post-injury. Despite remaining in VS or MCS for a median of 47 days, $20 \%$ of the entire cohort recovered all six target behaviors by the end of the observation period. Recovery rates were two to three times higher among patients who were within 10 weeks of injury at enrollment than in those who were 11-16 weeks post-injury. Recovery rates were approximately three times higher for patients in MCS (range $=40-57 \%$ ) than in those in VS (range $=11-23 \%$ ). Additionally, more than one third of the MCS subgroup recovered at least five target behaviors and more than half emerged from MCS within the 6 week interval.

Our findings comport with evidence from structural and functional neuroimaging studies showing differences in corticocortical and corticothalamic connectivity between patients in MCS and those in VS. Diffusion tensor imaging ${ }^{27}$ and resting state functional magnetic resonance imaging (fMRI) studies $^{28,29}$ have shown that the integrity of the frontoparietal association cortex, default mode network, and salience network reliably differentiate patients in MCS from those in VS. These observations suggest a graded relationship between network connectivity and level of consciousness and are consistent with the role of these regions in perceptual processing, attentional orienting, and vigilance. The degree of connectivity of two particular structures in the consciousness network - the posterior cingulate cortex and precuneus - has also been linked to subsequent behavioral recovery. ${ }^{28,30,31}$ Although the MCS diagnosis clearly conferred a more favorable prognosis for behavioral recovery, it is also notable that $14 \%$ of the VS subgroup recovered at least five of the six target behaviors and $20 \%$ were consistently following commands by the end of the 6 week interval.

We found a linear relationship between the number of behaviors recovered and degree of disability at follow-up. For each behavior recovered, the DRS score improved by nearly 2 points, resulting in a 12 point spread between patients who recovered all six behaviors and those who recovered none. This relationship highlights the importance of these six behaviors in functional recovery. The number of behaviors recovered is essentially a proxy for the degree of preservation of language and motor networks, which likely underlies the extent of functional improvement observed. ${ }^{27-29}$ 
Our findings also point to the critical role of language function in recovery. The proportion of patients who recovered all six target behaviors and the frequency of emergence from MCS were significantly higher in the MCS+ subgroup, and these improvements were associated with less disability on the DRS. Although the MCS- subgroup recovered more behaviors than the VS subgroup, outcomes were not substantially different at week 6 . These findings comport with the results of diffusion tractography ${ }^{32}$ and fluorodeoxyglucose (FDG)-positron emission tomography (PET) ${ }^{3}$ studies showing significantly reduced connectivity and diminished metabolic activity, respectively, between the left temporal cortex and the thalamus in patients in MCS- relative to those in MCS+. Taken together, our results support the proposed subcategorization of MCS, ${ }^{3}$ suggest that MCS+ may be a favorable early prognostic indicator, and underscore the important influence of language on functional outcome.

This study has several limitations. First, because our sample was composed of patients undergoing inpatient rehabilitation, there may have been a selection bias toward admitting those with more favorable prognoses. This seems unlikely, as patients in VS comprised more than one third of our sample, and none of the subjects were able to communicate reliably at enrollment. Second, we did not attempt to parse the effects of rehabilitation on outcome, leaving open the possibility that recovery was influenced by rehabilitation therapies. Although treatment effects cannot be ruled out, patients were receiving the usual care, and pharmacological interventions to accelerate recovery were prohibited. Third, stratifying the sample into subgroups by diagnosis and time post-injury limited the number of patients available for some analyses, reducing power and generalizability. A larger sample would have enabled comparison of outcomes across these substrata. This issue highlights the need for larger studies that can support more granular outcome assessment. Nonetheless, the subgroup findings and precision parameters reported here will support sample size calculations, and our results concerning the relationship between number of behaviors recovered and degree of disability can be used to establish a minimum clinically important difference for the DRS. ${ }^{33}$

Our findings suggest that one in five patients who remain in traumatic VS or MCS for at least 4 weeks will subsequently recover the ability to communicate, verbalize intelligibly, follow commands, recognize familiar stimuli, and utilize common objects. Nearly one third, including $11 \%$ of those in VS, will recover the ability to communicate reliably. Reliable communication is highly valued by family members, ${ }^{8}$ and is critically important when assessing pain, hunger, and thirst. These findings have three important clinical implications. First, clinicians involved in planning treatment should recognize that failure to emerge from traumatic DoC within 4 weeks of injury does not necessarily portend unfavorable outcome. Second, when engaged in prognostic counseling, clinicians should explain that behavioral signs of consciousness may not emerge until after the 1st month postinjury. Third, aggressive medical and rehabilitative interventions should be among the options considered for patients with traumatic DoC who are within 1 month of injury. Our results lend further support to the newly released DoC practice guidelines, which recommend that when clinicians are discussing prognosis with caregivers of patients with DoC who are within 28 days of injury, they must avoid statements that suggest that these patients have a universally poor prognosis. ${ }^{34}$ Although we did not monitor recovery beyond the 6 week interval, recent long-term outcome studies suggest that $\sim 20 \%$ of patients with prolonged DoC regain the capacity to live without in-home supervision within $2-5$ years of injury. ${ }^{9,35}$ Future research should investigate whether these target behaviors represent markers for eventual recovery of independence.

\section{Acknowledgments}

The authors thank Drs. Sabrina Taylor and Michael Bergin for reviewing the manuscript, and Thomas Quinn and Brendan Kelley for assisting with preparation of the data tables and for their carefully conducted analysis of data quality. The authors also thank all the investigators from the 11 original participating sites for their unswerving commitment to collaboration and rigor.

\section{Funding Information}

The contents of this manuscript were developed with grant funding by the National Institute on Disability, Independent Living and Rehabilitation Research (NIDILRR), formerly The National Institute on Disability and Rehabilitation Research (NIDRR). NIDILRR is a Center within the Administration for Community Living (ACL), Department of Health and Human Services (HHS). This article does not reflect the official policy or opinions of NIDILRR or HHS and does not constitute an endorsement by NIDILRR, HHS, or other components of the federal government. NIDILRR had no role in the design or conduct of the study; collection, management, analysis, or interpretation of the data; preparation, review, or approval of the manuscript; or the decision to submit the manuscript for publication. Dr. Joseph T. Giacino had full access to all the data in the study and takes responsibility for the integrity of the data and the accuracy of the data analysis. The data utilized in this manuscript and the authors' contributions were made possible by grant support from the National Institute on Disability and Rehabilitation Research (NIDRR), United States Department of Education (Award \# H133A031713: JFK-Johnson Rehabilitation Institute TBI Model System). Drs. Giacino, Hammond, and Sherer's contributions were also partially supported by awards from the National Institute on Disability, Independent Living and Rehabilitation Research (NIDILRR), Administration for Community Living (Award \#s 90DP0039, Spaulding-Harvard TBI Model System; 90DP0036, Indiana University TBI Model System; and 90DP0028, TIRR TBI Model System). Dr. Christoforou receives research support from the Department of Defense (grant \# W81XWH-14-2-0176). Dr. Long has received financial support through NIH grant N5065980, serves on the board of the Brain Injury Association of Pennsylvania, and is a recipient of the 2015 Council on Brain Injury (COBI) award. Dr. Sherer' research is funded by NIDILRR grants \# 90DP0028, 90RT5007, and 90DP0060 as well as NIH grant \# 1U01NS086090. Dr. Bagiella receives funding from National Heart, Lung, and Blood Institute (NHLBI) grant 5U01HL088942-08; NINDS grants 1R03NS07987501A1 and 6R37NS043209-14, AHRQ grant 5R01HS0229-03; NCATS grant 1UL1TR001433-01; NIGMS grant R25GM11123901A1; NHGRI grant 3U01HG007278-03S1; NIDDK grant 1R01DK102420-01A1; NCI grant 1R01CA163772-01A1; and the James S. McDonnell Foundation. The contents of this manuscript do not necessarily represent the policy of the U.S. Department of Health and Human Services, and endorsement by the federal government should not be assumed.

\section{Author Disclosure Statement}

Dr. Giacino is a member of the American Congress of Rehabilitation Medicine (ACRM), the Brain Injury Special Interest 
Group, and the Disorders of Consciousness Task Force; serves on a scientific advisory board for TBI Model Systems National Data and Statistical Center; has received funding for travel from the United States Department of Defense for a meeting related to the TBI Endpoint Development Project, the National Institute on Neurological Disorders and Stroke for the Traumatic Brain Injury Model Systems Project Directors meeting, and for a meeting related to the Transforming Research and Clinical Knowledge in Traumatic Brain Injury study, the National Institute on Disability, Independent Living and Rehabilitation Research, the American Academy of Physical Medicine and Rehabilitation, the One Mind Foundation, the James S. McDonnell Foundation for a meeting related to the Recovery of Consciousness After Severe Brain Injury study, the Barbara Epstein Foundation, and the International Brain Injury Association; has received a cash donation from the Epstein Foundation for a hospital clinical program that he directs and for serving on a team that provided clinical consultation services to an overseas patient who sustained severe brain injury; has served as an editor for the Journal of Head Trauma Rehabilitation; has received honoraria from the One Mind Foundation, Holy Cross Hospital (Surrey, UK), HealthSouth Braintree Hospital, Western Michigan Brain Injury Network, George Washington University Medical School, Association of Academic Physiatrists, Mayo Clinic, Kennedy-Krieger Institute, and Magill's Medical Guide; performs clinical procedures as $10 \%$ of his clinical effort in his role as Director of Spaulding Rehabilitation Network Disorders of Consciousness Program and neuroimaging as a principal investigator on two neuroimaging studies for $30 \%$ of his research effort; received financial support from the National Institutes of Health (NIH)-National Institute on Neurological Disorders and Stroke (NINDS) for Central Thalamic Stimulation for Traumatic Brain Injury, United States Department of Defense for TBI Endpoint Development Project, the Huperzine A for the Treatment of Cognitive, Mood and Functional Deficits After Moderate and Severe TBI study, the INjury and TRaumatic STress (INTRuST) Consortium Neuroimaging Acquisition and Archival study, the National Institute on Disability, Independent Living and Rehabilitation Research (NIDILRR) for the Spaulding Harvard - Traumatic Brain Injury Model System and for Multicenter Evaluation of Memory Remediation after Traumatic Brain Injury with Donepezil, NINDS for Transforming Research and Clinical Knowledge in Traumatic Brain Injury study, James S. McDonnell Foundation for Study of Recovery of Consciousness After Severe Brain Injury, Barbara Epstein Foundation, and the Spaulding Rehabilitation Hospital Department of Physical Medicine and Rehabilitation; and has acted as a witness with regard to a legal proceeding. Dr. Christoforou receives research support from the Department of Defense (grant \# W81XWH-14-2-0176). Dr. Hammond has consulted within the past 12 months for Avanir Pharmaceuticals, Inc. as a member of the PRISM II Steering Committee; has stock ownership in healthcare companies, including Abbvie Inc. SHS, Eli Lilly \& Co, GlaxoSmithKline PLC ADR, Exchange Traded Funds, and Mutual Funds; receives royalties from Lash Publishing and Demos Medical; and receives research support from the National Institute on Disability Independent Living and Rehabilitation Research. Dr. Long has received financial support through NIH grant N5065980, serves on the board of the Brain Injury Association of Pennsylvania; and is recipient of the 2015 COBI award. Dr. Maurer-Karattup reports no disclosures. Dr. Sherer is Associate Vice President for TIRR Memorial Hermann. His research is funded by NIDILRR grants \# 90DP0028, 90RT5007, and 90DP0060 as well as NIH grant \# 1U01NS086090. He serves on the editorial boards of Journal of Head Trauma Rehabilitation and Rehabilitation Psychology. In the past year he has received honoraria from the Council on Brain Injury, Center of Innovation on Disability and Rehabilitation Research, and the International Neuropsychological Society and has been reimbursed for travel expenses by the Council on Brain Injury, the North American Brain Injury Society, Center of Innovation on Disability and Rehabilitation Research, and the International Neuropsychological Society. He serves on the Advisory Board for the Center of Innovation on Disability and Rehabilitation Research at Malcolm Randall VA Medical Center.

Dr. Bagiella receives funding from NHLBI grant 5U01HL08894208; NINDS grants 1R03NS079875-01A1 and 6R37NS043209-14, AHRQ grant 5R01HS0229-03; NCATS grant 1UL1TR001433-01; NIGMS grant R25GM111239-01A1; NHGRI grant 3U01HG00727803S1; NIDDK grant 1R01DK102420-01A1; NCI grant 1R01CA163772-01A1; and the James S. McDonnell Foundation.

\section{Supplementary Material}

Supplementary Figure S1 Supplementary Table S1 Supplementary Table S2 Supplementary Table S3 Supplementary Table S4 Supplementary Table S5 Supplementary Table S6 Supplementary Table S7 Supplementary Table S8 Supplementary Table S9 Supplementary Table S10 Supplementary Table S11

\section{References}

1. The Multi-Society Task Force on PVS (1994). Medical aspects of the persistent vegetative state. N. Engl. J. Med. 330, 1499-1508.

2. Giacino, J.T., Ashwal, S., Childs, N., Cranford, R., Jennett, B., Katz, D.I., Kelly, J.P., Rosenberg, J.H., Whyte, J., Zafonte, R.D., and Zasler, N.D. (2002). The minimally conscious state: definition and diagnostic criteria. Neurology 58, 349-353.

3. Bruno, M.A., Majerus, S., Boly, M., Vanhaudenhuyse, A., Schnakers, C., Gosseries, O., Boveroux, P., Kirsch, M., Demertzi, A., Bernard, C., Hustinx, R., Moonen, G., and Laureys, S. (2012). Functional neuroanatomy underlying the clinical subcategorization of minimally conscious state patients. J. Neurol. 259, 1087-1098.

4. Schiff, N.D., Rodriguez-Moreno, D., Kamal, A., Kim, K.H., Giacino, J.T., Plum, F., and Hirsch, J. (2005) fMRI reveals large-scale network activation in minimally conscious patients. Neurology 64, 514-523.

5. Owen, A.M., Coleman, M.R., Boly, M., Davis, M.H., Laureys, S., and Pickard, J.D. (2006) Detecting awareness in the vegetative state. Science 313, 1402.

6. Whyte, J., Gosseries, O., Chervoneva, I., DiPasquale, M.C., Giacino, J., Kalmar, K., Katz, D.I., Novak, P., Long, D., Childs, N., Mercer, W., Maurer, P., and Eifert, B. (2009). Predictors of short-term outcome in brain-injured patients with disorders of consciousness. Prog. Brain. Res. 177, 63-72.

7. Noé, E., Olaya, J., Navarro, M.D., Noguera, P., Colomer, C., GarciaPanach, J., Rivero, S., Moliner, B., and Ferri, J. (2012). Behavioral recovery in disorders of consciousness: a prospective study with the Spanish version of the Coma Recovery Scale-Revised. Arch. Phys. Med. Rehabil. 93, 428-433.e12.

8. Giacino, J.T., Whyte, J., Bagiella, E., Kalmar, K., Childs, N., Khademi, A., Eifert, B., Long, D., Katz, D.I., Cho, S., Yablon, S.A., Luther, M., Hammond, F.M., Nordenbo, A., Novak, P., Mercer, W., Maurer-Karattup, P., and Sherer, M. (2012). Placebo-controlled trial of amantadine for severe traumatic brain injury. N. Engl. J. Med. 366, 819-826. 
9. Katz, D.I., Polyak, M., Coughlan, D., Nichols, M., and Roche, A. (2009). Natural history of recovery from brain injury after prolonged disorders of consciousness: outcome of patients admitted to inpatient rehabilitation with 1-4 year follow-up. Prog. Brain. Res. 177, 73-88.

10. Shiel, A., and Wilson, B.A. (2005). Can behaviours observed in the early stages of recovery after traumatic brain injury predict poor outcome? Neuropsychol. Rehabil. 15, 494-502.

11. Dolce, G., Lucca, L.F., Candelieri, A., Rogano, S., Pignolo, L., and Sannita, W.G. (2011). Visual pursuit in the severe disorder of consciousness. J. Neurotrauma 28, 1149-1154.

12. Steyerberg, E.W., Mushkudiani, N., Perel, P., Butcher, I., Lu, J., McHugh, G.S., Murray, G.D., Marmarou, A., Roberts, I., Habbema, J.D., and Maas, A. (2008). Predicting outcome after traumatic brain injury: development and international validation of prognostic scores based on admission characteristics. PLoS Med. 5, 1251-1261.

13. Jennett, B., and Bond, M. (1975). Assessment of outcome after severe brain damage. Lancet 1, 480-484.

14. Turgeon, A.F., Lauzier, F., Simard, J.F., Scales, D.C., Burns, K.E., Moore, L., Zygun, D.A., Bernard, F., Meade, M.O., Dung, T.C., Ratnapalan, M., Todd, S., Harlock, J., Fergusson, D.A., and Canadian Critical Care Trials Group (2011). Mortality associated with withdrawal of life-sustaining therapy for patients with severe traumatic brain injury: a Canadian multicentre cohort study. CMAJ 183, $1581-1518$.

15. Cuthbert, J.P., Harrison-Felix, C., Corrigan, J.D., Kreider, S., Bell, J.M., Coronado, V.G., and Whiteneck, G.G. (2015). Epidemiology of adults receiving acute inpatient rehabilitation for a primary diagnosis of traumatic brain injury in the United States. J. Head Trauma Rehabil. $30,122-135$.

16. Fins, J.J. (2003). Constructing an ethical stereotaxy for severe brain injury: balancing risks, benefits and access. Nat. Rev. Neurosci. 4, 323-327.

17. Fins, J. (2015). Rights Come to Mind: Brain Injury, Ethics, and the Struggle for Consciousness. Cambridge University Press: New York.

18. Quality Standards Subcommittee of the American Academy of Neurology (1995). Practice parameters: assessment and management of patients in the persistent vegetative state (summary statement). Neurology 45, 1015-1018.

19. Whyte, J., Katz, D., Long, D., DiPasquale, M., Polansky, M., Kalmar, K., Giacino, J., Childs, N., Mercer, W., Novak, P., Maurer, P., and Eifert, B. (2005). Predictors of outcome in prolonged posttraumatic disorders of consciousness and assessment of medication effects: a multicenter study. Arch. Phys. Med. Rehabil. 86, 453-462.

20. Giacino, J.T., Kalmar, K., and Whyte, J. (2004). The JFK Coma Recovery Scale-Revised: measurement characteristics and diagnostic utility. Arch. Phys. Med. Rehabil. 85, 2020-2029.

21. Rappaport, M., Hall, K.M., Hopkins, K., Belleza, T., and Cope, D.N. (1982). Disability rating scale for severe head trauma: coma to community. Arch. Phys. Med. Rehabil. 63, 118-123.

22. Schnakers, C., Vanhaudenhuyse, A., Giacino, J., Ventura, M., Boly, M., Majerus, S., Moonen, G., and Laureys, S. (2009). Diagnostic accuracy of the vegetative and minimally conscious state: clinical consensus versus standardized neurobehavioral assessment. BMC Neurol. 9, 35.

23. Seel R.T., Sherer, M., Whyte, J., Katz, D., Giacino, J.T., Shapiro, A., Hammond, F.M., Kalmar, K., Pape, T.L., Zafonte, R., Biester, R.C., Kaelin, D., Kean, J., and Zasler, N.D. (2010). Assessment scales for disorders of consciousness: evidence-based recommendations for clinical practice and research. Arch. Phys. Med. Rehabil. 91, 1795-1813.

24. Hicks, R., Giacino, J.T., Harrison-Felix, C., Manley, G., Valadka, A., and Wilde, E.A. (2013). Progress in developing Common Data Elements for Traumatic Brain Injury Research: Version 2 - The end of the beginning. J. Neurotrauma 30, 1852-1861.
25. Teasdale, G., and Jennett, B. (1974). Assessment of coma and impaired consciousness a practical scale. Lancet 2, 81-84.

26. Holm, S. (1979). A simple sequentially rejective multiple test procedure. Scand. J. Statist. 6, 65-70.

27. Fernández-Espejo, D., Soddu, A., Cruse, D., Palacios, E.M., Junque, C., Vanhaudenhuyse, A., Rivas, E., Newcombe, V., Menon, D.K., Pickard, J.D., Laureys, S., and Owen, A.M. (2012). A role for the default mode network in the bases of disorders of consciousness. Ann. Neurol. 72, 335-343.

28. Demertzi, A., Antonopoulos, G., Heine, L., Voss, H.U., Crone, J.S., de Los Angeles, C., Bahri, M.A., Di Perri, C., Vanhaudenhuyse, A., Charland-Verville, V., Kronbichler, M., Trinka, E., Phillips, C., Gomez, F., Tshibanda, L., Soddu, A., Schiff, N.D., Whitfield-Gabrieli, S., and Laureys, S. (2015). Intrinsic functional connectivity differentiates minimally conscious from unresponsive patients. Brain 138, 2619-2631.

29. Wu, X., Zou, Q., Hu, J., Tang, W., Mao, Y., Gao, L., Zhu, J., Jin, Y., Wu, X., Lu, L., Zhang, Y., Zhang, Y., Dai, Z., Gao, J.H., Weng, X., Zhou, L., Northoff, G., Giacino, J.T., He, Y., and Yang, Y. (2015). Intrinsic functional connectivity patterns predict consciousness level and recovery outcome in acquired brain injury. J. Neurosci. 35, $12,932-12,946$

30. Monti, M.M., Rosenberg, M., Finoia, P., Kamau, E., Pickard, J.D., and Owen, A.M. (2015). Thalamo-frontal connectivity mediates topdown cognitive functions in disorders of consciousness. Neurology $84,167-173$.

31. Stender, J., Gosseries, O., Bruno, M.A., Charland-Verville, V., Vanhaudenhuyse, A., Demertzi, A., Chatelle, C., Thonnard, M., Thibaut, A., Heine, L., Soddu, A., Boly, M., Schnakers, C., Gjedde, A., and Laureys, S. (2014). Diagnostic precision of PET imaging and functional MRI in disorders of consciousness: a clinical validation study. Lancet 384, 514-522.

32. Zheng, Z.S., Reggente, N., Lutkenhoff, E., Owen, A.M., and Monti, M.M. (2017). Disentangling disorders of consciousness: Insights from diffusion tensor imaging and machine learning. Hum. Brain. Mapp. $38,431-443$.

33. Jaeschke, R., Singer, J., and Guyatt, G.H. (1989). Measurement of health status. Ascertaining the minimal clinically important difference. Control. Clin. Trials. 10, 407-415.

34. Giacino, J.T., Katz, D.I., Schiff, N.D., Whyte, J., Ashman, E., Ashwal, S., Barbano, R.L., Hammond, F., Laureys, S., Ling, G.S.F., NakaseRichardson, R., Seel, R.T., Yablon, S.A., Getchius, T.G., Gronseth, G., and Armstrong, M.J. (2018). Practice guideline recommendations summary: Disorders of Consciousness, Report of the Guideline Development, Dissemination, and Implementation Subcommittee of the American Academy of Neurology; the American Congress of Rehabilitation Medicine; and the National Institute on Disability, Independent Living and Rehabilitation Research. Neurology 91, 450-460.

35. Nakase-Richardson, R., Whyte, J., Giacino, J.T., Pavawalla, S., Barnett, S.D., Yablon, S.A., Sherer, M., Kalmar, K., Hammond, F.M., Greenwald, B., Horn, L.J., Seel, R., McCarthy, M., Tran, J., and Walker, W.C. (2011). Longitudinal outcome of patients with disordered consciousness in the NIDRR TBI model systems programs. J. Neurotrauma 29, 59-65.

Address correspondence to: Joseph T. Giacino, PhD

Department of Physical Medicine and Rehabilitation Spaulding Rehabilitation Hospital 300 First Avenue

Charlestown, MA 02129

E-mail: jgiacino@mgh.harvard.edu 